\title{
A ring closing metathesis strategy for carbapyranosides of xylose and
}

\author{
arabinose
}

\author{
Clayton E. Mattis and David R. Mootoo* \\ Department of Chemistry, Hunter College and The Graduate Center \\ of the City University of New York, New York, NY 10016
}

\begin{abstract}
The synthesis of $\beta$-carba- xylo and arabino pyranosides of cholestanol is described. The synthetic strategy, which is analogous to the Postema approach to $C$-glycosides, centers on the ring closing metathesis of an enol ether-alkene precursor to give a cyclic enol ether that is elaborated to a carba-pyranoside via hydroboration-oxidation on the olefin. The method, which is attractive for its modularity and stereoselectivity, may find wider applications to carbahexopyranosides and other complex cycloalkyl ether frameworks.
\end{abstract}

Keywords: glycomimetic, carbasugar, cycloalkyl ether, arabinose, xylose, RCM

Chemical compounds studied in this article

Methyl 2,3-O-isopropylidene-beta-D-ribofuranoside (PubChem CID: 96666); Methyl alpha-Darabinofuranoside; (PubChem CID: 11389582; cholestanol (PubChem CID: 6665); Methyl

(triphenylphosphoranylidene)acetate (PubChem CID: 17453); Tebbe reagent (PubChem CID: 91617563);

Grubbs catalyst 2nd generation (PubChem CID: 11147261); Dimethyl sulfide borane (PubChem CID: 9833925)

\section{Introduction}

Sugar residues comprise the structures of a variety of bioactive natural products and invariably, impact on potency and, or specificity. ${ }^{1,2,3,4}$ Thus, glycodiversification is a popular strategy in the development of carbohydrate-based therapeutics. ${ }^{5,6,7,8}$ In this context, nonhydrolyzable sugar analogues such as carbasugars, in which the ring oxygen is replaced with a " $\mathrm{CH}_{2}$ ", have attracted attention as potentially metabolically stable therapeutic agents and for mechanistic studies. ${ }^{10}$ The nuanced conformational properties of carbasugars relative 
to their parent $O$-glycosides are of additional relevance to structure activity studies. ${ }^{11}$ Consequently there is much interest in the synthesis and properties of carbasugars. While several methods have been developed for carbasugars in which the pseudo sugar ring is linked to relatively simple alcohol segments or to the primary alcohol oxygen of a sugar, structures with more complex alcohol segments are not as easily accessible because of the challenges associated with fabricating the pseudoglycosidic ether bond. ${ }^{10,12,13,14} \mathrm{We}$ envisaged a RCM based approach to carbasugars that may address this issue and which has further appeal because of its modularity. This strategy is illustrated herein in the synthesis of $\beta$-carba- arabino and xylo pyranosides of cholestanol. We were drawn to these frameworks because of the existence of the parent sugars in several antitumor steroidal and triterpenoid saponins, of which OSW-1 1, is a notable example (Figure 1). ${ }^{15,16.17,18} \beta$-Xylopyranosides also comprise the capsular polysaccharide of fungal pathogens associated with AIDS. Carbaxylosides thereof may be of interest to vaccine development in this area. ${ }^{19}$

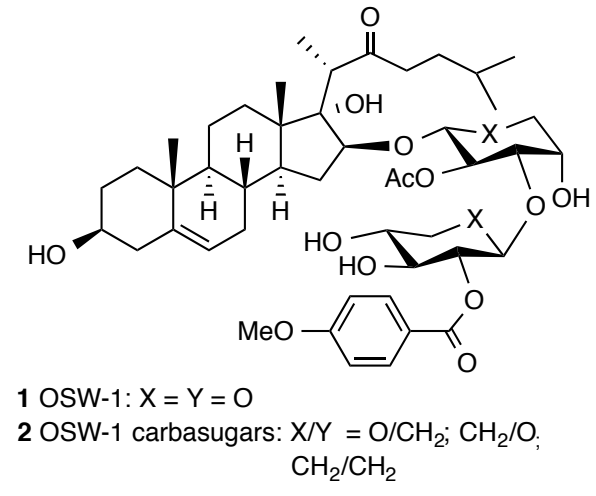

Figure 1. OSW-1 and carbasugar analogues

Our approach builds on the C-glycoside synthesis from the Postema group, in which the pivotal reaction is the RCM on an enol ether - alkene 5 to give the C1-substituted glycal 4 (Scheme 1). ${ }^{20}$ Stereoselective hydroboration-oxidation on 5 leads to the1,2-trans / 2,3-trans 
C-glycoside 3. An attractive feature of this strategy is the modular assembly from "glycone" and "aglycone" precursors 6 and 7. Through the use of C-branched sugar acids, this method has been applied to C-di- and higher order $C$-glycosides. An analogous strategy for carbasugars calls for a RCM on an enolether alkene $\mathbf{1 0}$ to give the cyclic enol ether $\mathbf{9}$, which differs from 4, the corresponding enol ether in the C-glycoside synthesis, in that the enol ether oxygen is exocyclic and not endocyclic. However, while RCM's on enol ether alkenes like $\mathbf{5}$ have been successful on a variety of highly substituted substrates, to the best of our knowledge, RCMs on variants like 10, in which the ether oxygen is exocyclic to the eventual ring, have only been tested on silyl or simple alkyl enol ethers. ${ }^{21,22,23,24,25}$

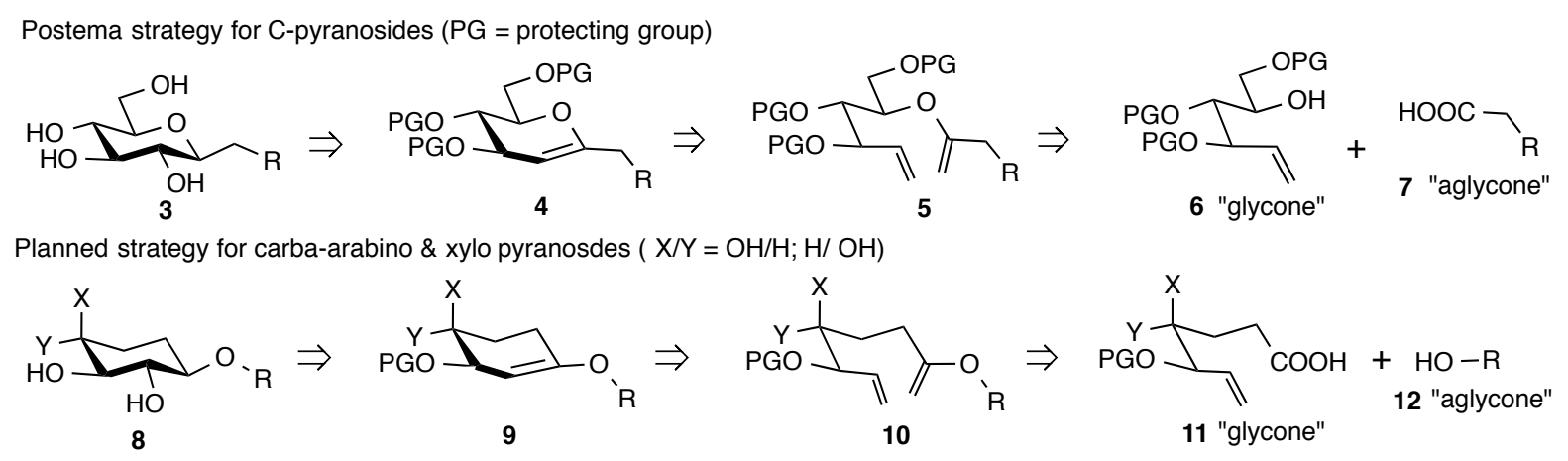

\section{Scheme 1. RCM strategies to $\mathrm{C}$ - and carba- pyranosides}

\section{Results and discussion}

Synthesis of "glycone" segments. The unsaturated acid precursor $\mathbf{1 5}$ for carbaarabinoses was obtained by hydrolysis of the known ester 14, which in turn was prepared from 5-deoxy-5-iodo-D-ribo-furanoside 13, via a known procedure (Scheme 2) ${ }^{26}$ The carbaxyloside precursor 19, which was previously prepared from L-tartaric, was prepared here via a more concise route, using a strategy similar to that used for $14 .{ }^{27}$ Thus, zinc mediated 
reductive opening on the 5-deoxy-5-iodo-D-arabinofuranoside $\mathbf{1 6}$ afforded enal $\mathbf{1 7} .^{28,29}$ Treatment of $\mathbf{1 7}$ with methyl(triphenylphosphoranylidene)acetate provided $\mathbf{1 8}$ as a single $E$ isomer. Selective hydrogenation of the conjugated alkene followed by hydrolysis of the ester led to 19.
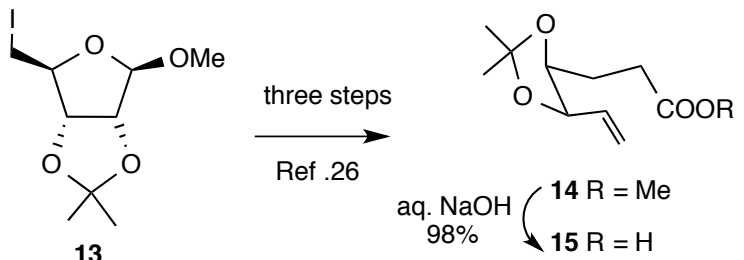

13

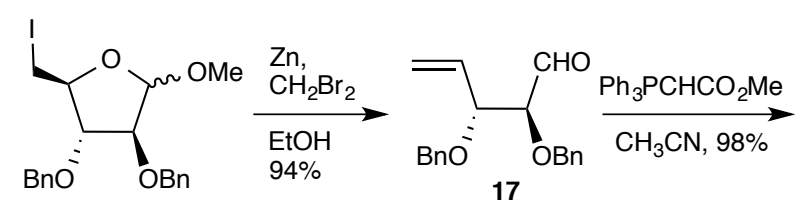

16

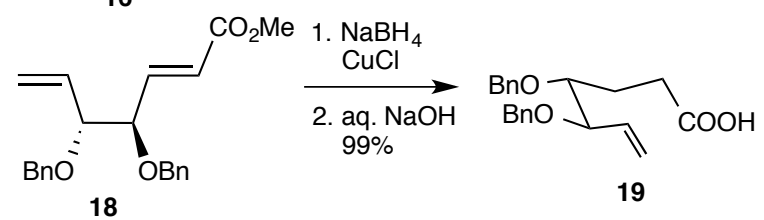

\section{Scheme 2. Synthesis of "glycone" precursors}

The feasibility of the key RCM reaction was tested using cholestanol $\mathbf{2 0}$ as a model steroidal segment (Scheme 3). Accordingly, DCC promoted esterification of $\mathbf{2 0}$ and alkenoic acids $\mathbf{1 5}$ and 19 produced esters 21 and 25 in 98 and 90\% yields respectively. Next, olefination on 21 and 25 using the Tebbe and Takai reagents, afforded the respective enol ethers 22 and $\mathbf{2 6}$ in 70 and $66 \%$ yield..$^{30,31}$ These materials were sensitive to acid and silica gel purification required the presence of triethylamine in the mobile phase. Treatment of $\mathbf{2 2}$ and $\mathbf{2 6}$ with 10 mole \% Grubbs II catalyst in dichloromethane at $60{ }^{\circ} \mathrm{C}$ led to the cyclic enol ethers 23 and 27 in 75 and $80 \%$ yields respectively. Finally, a hydroboration - oxidation sequence on $\mathbf{2 3}$ and 27 afforded the $\beta$-carba- arabinoside and xyloside $\mathbf{2 4}$ and $\mathbf{2 8}$ respectively, as the only observed diastereomers, in 80 and $63 \%$ yields. The stereochemistry of $\mathbf{2 4}$ and $\mathbf{2 8}$ was 
assigned from $1 \mathrm{H}$ NMR analysis of their acetates $24-O A c\left(\mathrm{~J}_{1}, 2^{\prime},=9.5, \mathrm{~J}_{2}, 3^{\prime},=9.9, \mathrm{~J}_{3}, 4^{\prime},=\right.$ $5.1 \mathrm{~Hz})$ and 28-OAc $\left(\mathrm{J}_{1^{\prime}, 2^{\prime}}=\mathrm{J}_{2^{\prime}, 3^{\prime}}=\mathrm{J}_{3^{\prime}, 4^{\prime}}=9.5-9.6 \mathrm{~Hz}\right.$, see supporting information for selected ${ }^{1} \mathrm{HNMR}$ assignments). NOEs between $\mathrm{H} 1$ '-H3' and $\mathrm{H} 2$ '-H4' supported the structure of 28-OAc.

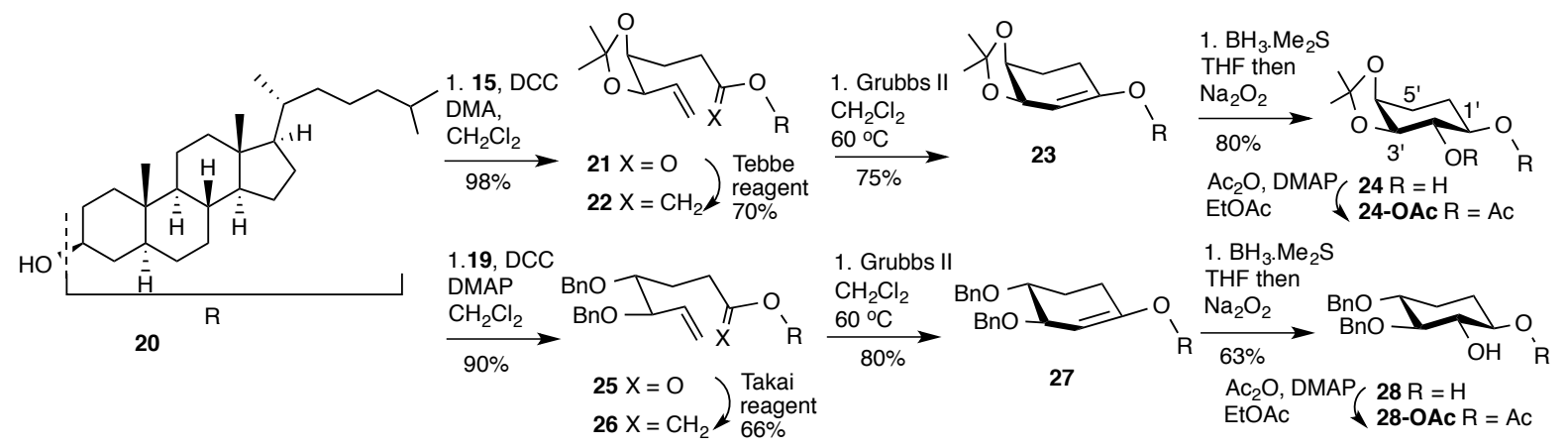

\section{Scheme 3. Synthesis of carba-3 $\beta$-cholestanyl pentopyranosides}

\section{Conclusion}

In summary this RCM approach to cholestanol carba-pentopyranosides $\mathbf{2 4}$ and $\mathbf{2 8}$ illustrates a potentially general strategy for the synthesis of $\beta$-carba- arabino and xylo- pyranosides with complex aglycone segments. The methodology, which constitutes a synthesis of stereochemically complex cycloalkyl-ethers, is attractive for its modularity and stereoselectivity. The synthesis of carba-hexopyranosides is an obvious direction for future study and application to other groups of cycloalkyl ether frameworks is also envisaged.

\subsection{Synthesis - General}

Solvents were purified by standard procedures or used from commercial sources as appropriate. Petroleum ether refers to the fraction of petroleum ether boiling between 40 and $60{ }^{\circ} \mathrm{C}$. Ether refers to diethyl ether. Unless otherwise stated thin layer chromatography (TLC) 
was done on $0.25 \mathrm{~mm}$ thick precoated silica gel 60 (HF-254, Whatman) aluminium sheets and flash column chromatography (FCC) was performed using Kieselgel 60 (32-63 mesh, Scientific Adsorbents). Elution for FCC usually employed a stepwise solvent polarity gradient, correlated with TLC mobility. Chromatograms were observed under UV (short and long wavelength) light, and/or were visualized by heating plates that were dipped in a solution of ammonium (VI) molybdate tetrahydrate (12.5 g) and cerium (IV) sulfate tetrahydrate $(5.0 \mathrm{~g})$ in $10 \%$ aqueous sulphuric acid $(500 \mathrm{~mL})$, or a solution of $20 \%$ sulfuric acid in ethanol. NMR spectra were recorded using Varian Unity Plus 500 and Bruker Ultra Shield Plus $600 \mathrm{MHz}$ instruments, in $\mathrm{CDCl}_{3}$ or $\mathrm{C}_{6} \mathrm{D}_{6}$ solutions with residual $\mathrm{CHCl}_{3}$ or $\mathrm{C}_{6} \mathrm{H}_{6}$ as internal standard $\left(\delta_{\mathrm{H}} 7.27,7.16\right.$ and $\left.\delta_{\mathrm{C}} 77.2,128.4 \mathrm{ppm}\right)$. Optical rotations $\left([\alpha]_{\mathrm{D}}\right.$ were recorded using a Jasco P-1020 polarimeter and are given in units of $10^{-1} \mathrm{degcm}^{2} \mathrm{~g}$ at $589 \mathrm{~nm}$ (sodium D-line). Chemical shifts are quoted in ppm relative to tetramethysilane $\left(\delta_{\mathrm{H}} 0.00\right)$ and coupling constants (J) are given in Hertz. First order approximations are employed throughout. High resolution mass spectrometry was performed on Ultima Micromass Q-TOF or Waters Micromass LCT Premier mass spectrometers.

\section{2 (4S, 5R)-4,5-O-isopropylidene-hept-6-enoic acid (15)}

Methyl ester $14(1.21 \mathrm{~g}, 5.65 \mathrm{mmol})$ was dissolved in 5:1 THF: $\mathrm{H}_{2} \mathrm{O}(12 \mathrm{~mL})$ and $3 \mathrm{~N}$ $\mathrm{NaOH}(6 \mathrm{~mL})$ was added. The mixture was stirred vigorously for $16 \mathrm{~h}$, then brought to $\mathrm{pH} 5$ by the addition of $1 \mathrm{~N} \mathrm{HCl}(15 \mathrm{~mL})$, and extracted with EtOAc. The combined organic phase was washed with brine, dried $\left(\mathrm{Na}_{2} \mathrm{SO}_{4}\right)$, and concentrated in vacuo. FCC of the residual oil afforded 15 (1.12 g, 98\%): $\mathrm{R}_{\mathrm{f}}=0.4$ (20\% EtOAc: petroleum ether); ${ }^{1} \mathrm{H}$ NMR (600 MHz, $\left.\mathrm{CDCl}_{3}\right) \delta 1.38(\mathrm{~s}, 3 \mathrm{H}), 1.49(\mathrm{~s}, 3 \mathrm{H}), 1.76(\mathrm{~m}, 2 \mathrm{H}), 2.46(\mathrm{~m}, 1 \mathrm{H}), 2.55(\mathrm{~m}, 1 \mathrm{H}), 4.19(\mathrm{~m}, 1 \mathrm{H})$ 
$4.57(\mathrm{t}, 1 \mathrm{H}, \mathrm{J}=6.9 \mathrm{~Hz}), 5.29(\mathrm{~d}, 1 \mathrm{H}, \mathrm{J}=10.4 \mathrm{~Hz}), 5.37(\mathrm{~d}, 1 \mathrm{H}, \mathrm{J}=17.2 \mathrm{~Hz}), 5.85(\mathrm{~m}, 1 \mathrm{H})$, 11.51 (bs, $1 \mathrm{H}) ;{ }^{13} \mathrm{C}$ NMR $\left(150 \mathrm{MHz}, \mathrm{CDCl}_{3}\right) \delta 25.6,25.8,28.1,30.6,79.5,108.6,118.9$, 133.6, 179.5; ESIHRMS (M+H) ${ }^{-}$calculated for $\mathrm{C}_{10} \mathrm{H}_{15} \mathrm{O}_{4}$ 199.0970, found 199.0977 .

\section{$4.3 \quad(2 S, 3 R)-2,3-b i s($ benzyloxy)pent-4-enal (17)}

Activated zinc dust $(5.14 \mathrm{~g}, 79.7 \mathrm{mmol})$ was added to a solution of $\mathbf{1 6}(3.62 \mathrm{~g}, 7.97$ $\mathrm{mmol})$ in $\mathrm{EtOH}(30 \mathrm{~mL})$ 1,2-Dibromoethane $(0.2 \mathrm{~mL})$ was introduced and the mixture stirred for $2 \mathrm{~h}$ then filtered over a bed of Celite. The filtrate was concentrated in vacuo and the residue purified by FCC to give $\mathbf{1 7}(2.22 \mathrm{~g}, 94 \%)$ as a colorless oil: $\mathrm{R}_{\mathrm{f}}=0.6(20 \%$ EtOAc:

petroleum ether); ${ }^{1} \mathrm{H}$ NMR (500 MHz, $\left.\mathrm{CDCl}_{3}\right) \delta 3.76(\mathrm{dd}, 1 \mathrm{H}, \mathrm{J}=1.5,4.5 \mathrm{~Hz}), 4.10(\mathrm{dd}, 1 \mathrm{H}$, $\mathrm{J}=4.5,8.0 \mathrm{~Hz}), 4.48(\mathrm{ABq}, 2 \mathrm{H}, \Delta \delta=0.36 \mathrm{ppm}, \mathrm{J}=12.0 \mathrm{~Hz}), 4.62(\mathrm{ABq}, 2 \mathrm{H}, \Delta \delta=0.11$ ppm, $\mathrm{J}=12.0 \mathrm{~Hz}), 5.29(\mathrm{~m}, 2 \mathrm{H}), 5.87(\mathrm{~m}, 1 \mathrm{H}), 7.19-7.29(\mathrm{~m}, 10 \mathrm{H}), 9.6(\mathrm{~s}, 1 \mathrm{H}) ;{ }^{13} \mathrm{C} \mathrm{NMR}$ $\left(125 \mathrm{MHz}, \mathrm{CDCl}_{3}\right) \delta$ 70.7, 73.5, 79.9, 85.2, 119.9, 127.8, 127.9, 128.1, 128.2, 128.4, 128.5, 133.8, 137.1, 137.5, 202.6; ESILRMS $(\mathrm{M}+\mathrm{Na})^{+}$calculated for $\mathrm{C}_{19} \mathrm{H}_{20} \mathrm{O}_{3} \mathrm{Na}$ 319.1, found 319.1 .

\subsection{Methyl $(4 R, 5 R, E)-4,5$-bis(benzyloxy)hepta-2,6-dienoate (18)}

A mixture of $17(2.0 \mathrm{~g}, 6.76 \mathrm{mmol})$ and $\mathrm{Ph}_{3} \mathrm{P}=\mathrm{CHCO}_{2} \mathrm{Me}(4.52 \mathrm{~g}, 13.52 \mathrm{mmol})$ in dry $\mathrm{CH}_{3} \mathrm{CN}(40 \mathrm{~mL})$ was heated at reflux for $2 \mathrm{~h}$. After cooling to $\mathrm{rt}$, the mixture was filtered and the filtrate concentrated under reduced pressure. FCC of the residue afforded $\mathbf{1 8}$ (2.38 g, 98\%) as a colorless oil: $\mathrm{R}_{\mathrm{f}}=0.8\left(20 \%\right.$ EtOAc: petroleum ether); ${ }^{1} \mathrm{H}$ NMR (500 MHz, $\left.\mathrm{CDCl}_{3}\right)$ $\delta 3.78(\mathrm{~s}, 3 \mathrm{H}), 3.96(\mathrm{dd}, 1 \mathrm{H}, \mathrm{J}=5.5,6.5 \mathrm{~Hz}), 4.14(\mathrm{dt}, 1 \mathrm{H}, \mathrm{J}=1.0,5.5 \mathrm{~Hz}), 4.45(\operatorname{app~d}, 1 \mathrm{H}$, $\mathrm{J}=12.0 \mathrm{~Hz}), 4.54(\operatorname{app~d}, 1 \mathrm{H}, \mathrm{J}=12.0 \mathrm{~Hz}), 4.67(\operatorname{app~d}, 2 \mathrm{H}, \mathrm{J}=12.0 \mathrm{~Hz}), 5.35(\mathrm{~m}, 2 \mathrm{H}), 5.80$ 
(m, 1H), $6.11(\mathrm{dd}, 1 \mathrm{H}, \mathrm{J}=1.5,16.0 \mathrm{~Hz}), 6.96(\mathrm{dd}, 1 \mathrm{H}, \mathrm{J}=6.0,16.0 \mathrm{~Hz}), 7.30-7.35(\mathrm{~m}, 10 \mathrm{H})$;

${ }^{13} \mathrm{C}$ NMR $\left(125 \mathrm{MHz}, \mathrm{CDCl}_{3}\right) \delta 51.6,70.7,71.9,79.9,81.7,119.6,122.8,127.6,127.7,128.3$, $128.4,134.3,137.9,138.2,145.1,166.5$; ESILRMS $(\mathrm{M}+\mathrm{Na})^{+}$calculated for $\mathrm{C}_{22} \mathrm{H}_{24} \mathrm{O}_{4} \mathrm{Na}$ 375.2 , found 375.2 .

\section{$4.5 \quad(4 R, 5 R)-4,5$-bis(benzyloxy)hept-6-enoic acid (19)}

Conjugated ester 18 (1.20 g, $3.40 \mathrm{mmol})$ was dissolved in $\mathrm{MeOH}(30 \mathrm{~mL})$ and $\mathrm{CuCl}$ (40 mg, $0.34 \mathrm{mmol})$ was added and the reaction mixture cooled to $-78{ }^{\circ} \mathrm{C} . \mathrm{NaBH}_{4}(646 \mathrm{mg}$, $17.1 \mathrm{mmol}$ ) was then added in one portion to the reaction. The brown slurry was stirred vigorously at $-78{ }^{\circ} \mathrm{C}$ until the color changed from brown to black over a period of $2 \mathrm{~h}$. The reaction was then slowly warmed to rt, filtered and concentrated in vacuo to give a crude oil. FCC of the residual oil gave the dihydroderivative as a pale yellow oil $(1.18 \mathrm{~g}, 99 \%): \mathrm{R}_{\mathrm{f}}=$ 0.7 (10\% EtOAc: petroleum ether). ${ }^{1} \mathrm{H}$ NMR $\left(500 \mathrm{MHz}, \mathrm{CDCl}_{3}\right) \delta 1.77(\mathrm{~m}, 1 \mathrm{H}), 1.96(\mathrm{~m}$, 1H), $2.40(\mathrm{~m}, 1 \mathrm{H}), 3.55(\mathrm{~m}, 1 \mathrm{H}), 3.63(\mathrm{~s}, 3 \mathrm{H}), 3.94(\mathrm{t}, 1 \mathrm{H}, \mathrm{J}=6.7 \mathrm{~Hz}), 4.43(\operatorname{app} \mathrm{d}, 1 \mathrm{H}, \mathrm{J}=$ $12.0 \mathrm{~Hz}), 4.55(\operatorname{app} \mathrm{d}, 1 \mathrm{H}, \mathrm{J}=11.4 \mathrm{~Hz}), 4.67(\operatorname{app} \mathrm{d}, 1 \mathrm{H}, \mathrm{J}=12.0 \mathrm{~Hz}), 4.78(\operatorname{app} \mathrm{d}, 1 \mathrm{H}, \mathrm{J}=$ $12.0 \mathrm{~Hz}), 5.36(\mathrm{~m}, 2 \mathrm{H}), 5.85(\mathrm{~m}, 1 \mathrm{H}), 7.29-7.36(\mathrm{~m}, 10 \mathrm{H}) ;{ }^{13} \mathrm{C} \mathrm{NMR}\left(125 \mathrm{MHz}, \mathrm{CDCl}_{3}\right) \delta$ $26.2,30.2,51.5,70.6,73.3,80.0,82.6,119.1,127.5,127.6,127.7,128.0,128.3,128.3,128.4$, 135.0, 138.5, 138.6, 174.1; ESILRMS $(\mathrm{M}+\mathrm{Na})^{+}$calculated for $\mathrm{C}_{22} \mathrm{H}_{26} \mathrm{O}_{4} \mathrm{Na}$ 377.2, found 377.2.

The material from the previous step $(1.10 \mathrm{~g}, 3.11 \mathrm{mmol})$ was transformed to $19(1.05 \mathrm{~g}, 99 \%)$ following the hydrolysis procedure that was used for 15. For 19: $R_{f}=0.6(30 \%$ EtOAc: petroleum ether). ${ }^{1} \mathrm{H}$ NMR (500 MHz, $\left.\mathrm{CDCl}_{3}\right) \delta 1.67(\mathrm{~m}, 1 \mathrm{H}), 1.86(\mathrm{~m}, 1 \mathrm{H}), 2.33(\mathrm{~m}, 2 \mathrm{H})$, $3.48(\mathrm{~m}, 1 \mathrm{H}), 3.85(\mathrm{t}, 1 \mathrm{H}, \mathrm{J}=6.7 \mathrm{~Hz}), 4.45(\mathrm{ABq}, 2 \mathrm{H}, \Delta \delta=0.36 \mathrm{ppm}, \mathrm{J}=12.0 \mathrm{~Hz}), 4.58$ 
$(\mathrm{ABq}, 2 \mathrm{H}, \Delta \delta=0.23 \mathrm{ppm}, \mathrm{J}=11.5 \mathrm{~Hz}), 5.27(\mathrm{~m}, 2 \mathrm{H}), 5.74(\mathrm{~m}, 1 \mathrm{H}), 7.20-7.26(\mathrm{~m}, 10 \mathrm{H}) ;{ }^{13} \mathrm{C}$ NMR $\left(125 \mathrm{MHz}, \mathrm{CDCl}_{3}\right) \delta$ 23.6, 27.8, 68.3, 71.1, 77.6, 80.2, 117.0, 125.3, 125.4, 125.5, 125.8, 126.1, 132.5, 136.0, 136.1, 175.9; ESILRMS (M-H) ${ }^{-}$calculated for $\mathrm{C}_{21} \mathrm{H}_{23} \mathrm{O}_{4}$ 339.17, found 339.15 .

\subsection{3ß-Cholestanyl $(4 S, 5 R)-4,5-O$-isopropylidene-hep-6-enoate (21)}

Cholestanol 21 (972 mg, $2.50 \mathrm{mmol}$ ) was added to a mixture of 15 (500 mg, $2.50 \mathrm{mmol})$, DCC (516 mg, $2.50 \mathrm{mmol})$ and DMAP (31 mg, $0.25 \mathrm{mmol})$ in DCM (10 mL). The reaction mixture was stirred for $1 \mathrm{~h}$ then diluted with ether and filtered. The filtrate was successively washed with $0.1 \mathrm{~N}$ aqueous $\mathrm{HCl}$ and brine, dried $\left(\mathrm{Na}_{2} \mathrm{SO}_{4}\right)$, filtered, and evaporated in vacuo. FCC of the residue gave ester 21 (1.40 g, 98\%): $\mathrm{R}_{\mathrm{f}}=0.7$ (10\% EtOAc: petroleum ether); ${ }^{1} \mathrm{H}$ NMR (600 MHz, $\left.\mathrm{C}_{6} \mathrm{D}_{6}\right) \delta 0.47(\mathrm{t}, 1 \mathrm{H}, \mathrm{J}=11.8 \mathrm{~Hz}), 0.65(\mathrm{~s}, 3 \mathrm{H}, \mathrm{H}-18)$, 0.68 (s, 3H, H-19), $0.78-0.95$ (m, buried 5H), 0.93 (bd, 6H, J = 6.5 Hz, H-26, 27), 1.02 (d, $3 \mathrm{H}, \mathrm{J}=6.4 \mathrm{~Hz}), 1.15-1.63(\mathrm{~m}, 22 \mathrm{H}), 1.28(\mathrm{~s}$, buried, 3H), $1.47(\mathrm{~s}$, buried, 3H), $1.68(\mathrm{~m}$, 1H), $1.74(\mathrm{~m}, 1 \mathrm{H}), 1.86(\mathrm{~m}, 2 \mathrm{H}), 1.98(\mathrm{bd}, 1 \mathrm{H}, \mathrm{J}=12.8 \mathrm{~Hz}), 2.45(\mathrm{~m}, 1 \mathrm{H}), 2.55(\mathrm{~m}, 1 \mathrm{H}), 4.01$ (m, 1H), $4.33(\mathrm{t}, 1 \mathrm{H}, \mathrm{J}=6.7 \mathrm{~Hz}), 4.93(\mathrm{~m}, 1 \mathrm{H}), 5.00(\mathrm{~d}, 1 \mathrm{H}, \mathrm{J}=10.4 \mathrm{~Hz}), 5.18(\mathrm{~d}, 1 \mathrm{H}, \mathrm{J}=$

$17.0 \mathrm{~Hz}), 5.70(\mathrm{~m}, 1 \mathrm{H}) ;{ }^{13} \mathrm{C} \mathrm{NMR}\left(150 \mathrm{MHz}, \mathrm{C}_{6} \mathrm{D}_{6}\right) \delta 12.1$ (two peaks), 18.8, 21.3, 22.6, $22.8,24.1,24.3,25.5,26.6,27.8,28.2,28.5,28.7,31.4,32.0,34.2,34.3,35.4,35.5,36.0$, $36.5,36.7,39.7,40.2,42.7,44.5,54.1,56.4,56.5,73.4,77.3,79.4,108.2,117.3,134.7$, 172.2; ESIHRMS $(\mathrm{M}+\mathrm{H})^{+}$calculated for $\mathrm{C}_{37} \mathrm{H}_{63} \mathrm{O}_{4} 571.4726$, found 571.4717.

\section{7 (3R)-((5S,6R)-5,6-O-isopropylidene-octa-1,7-dien-2-yl)oxy)-cholestane (22)}

Tebbe reagent (5.71 $\mathrm{mL}, 0.5 \mathrm{M}$ in THF) was added under an argon atmosphere, at -78 
${ }^{\mathrm{O}} \mathrm{C}$, to a mixture of $21(200 \mathrm{~g}, 0.35 \mathrm{mmol})$, pyridine $(0.10 \mathrm{~mL})$ and $3: 1$ anhydrous toluene:THF $(6 \mathrm{~mL})$. The reaction mixture was warmed to $\mathrm{rt}$, maintained at this temperature for $1 \mathrm{~h}$, then poured into $1 \mathrm{~N}$ aqueous $\mathrm{NaOH}$ at $0{ }^{\circ} \mathrm{C}$. The resulting suspension extracted with ether and the combined organic phase washed with brine, dried $\left(\mathrm{Na}_{2} \mathrm{SO}_{4}\right)$, filtered and concentrated in vacuo. FCC of the crude material over basic alumina afforded 22 (140 mg, $70 \%$ based on recovered starting material) as light yellow oil: $\mathrm{R}_{\mathrm{f}}=0.7$ (basic alumina, $10 \%$ EtOAc: petroleum ether: $2 \%$ TEA); ${ }^{1} \mathrm{H}$ NMR $\left(500 \mathrm{MHz}, \mathrm{C}_{6} \mathrm{D}_{6}\right) \delta 0.64(\mathrm{dt}, 1 \mathrm{H}, \mathrm{J}=3.8 .11 .0$ Hz), $0.78(\mathrm{~s}, 3 \mathrm{H}), 0.82(\mathrm{~s}, 3 \mathrm{H}), 0.85-1.10(\mathrm{~m}, 5 \mathrm{H}), 1.06(\mathrm{~d}, 6 \mathrm{H}, \mathrm{J}=6.6 \mathrm{~Hz}), 1.15(\mathrm{~d}, 3 \mathrm{H}, \mathrm{J}=$ $6.6 \mathrm{~Hz}), 1.20-1.80(\mathrm{~m}, 19 \mathrm{H}), 1.42$ (s, buried, 3H), 1.62 (s, buried, 3H), $1.74(\mathrm{~m}, 2 \mathrm{H}), 1.90$ (m, 2H), $2.01(\mathrm{~m}, 2 \mathrm{H}), 2.13(\mathrm{~m}, 2 \mathrm{H}), 2.43(\mathrm{~m}, 1 \mathrm{H}), 2.64 \mathrm{~m}, 1 \mathrm{H}), 4.08(\mathrm{~m}, 1 \mathrm{H}), 4.17(\mathrm{~s}, 1 \mathrm{H})$ $4.23(\mathrm{~m}, 1 \mathrm{H}), 4.25(\mathrm{~s}, 1 \mathrm{H}), 4.51(\mathrm{t}, 1 \mathrm{H}, \mathrm{J}=6.4 \mathrm{~Hz}), 5.15(\mathrm{~d}, 1 \mathrm{H}, \mathrm{J}=10.4 \mathrm{~Hz}), 5.32(\mathrm{bd}, 1 \mathrm{H}, \mathrm{J}$ $=16.6 \mathrm{~Hz}), 5.92(\mathrm{~m}, 1 \mathrm{H}) ;{ }^{13} \mathrm{C} \mathrm{NMR}\left(125 \mathrm{MHz}, \mathrm{C}_{6} \mathrm{D}_{6}\right) \delta 12.7$ (two peaks), 19.4, 21.9, 23.1, $23.4,24.7,24.9,26.2,28.3,28.8,28.9,29.0,29.3,29.6,32.8,33.4,34.8,36.0,36.2,36.6$, 37.0, 37.4, 40.3, 40.8, 43.3, 45.2, 54.9, 57.1 (two peaks), 75.8, 78.3, 80.3, 81.9, 108.6, 117.6, 135.9, 161.8; ESIHRMS $(\mathrm{M}+\mathrm{H})^{+}$calculated for $\mathrm{C}_{38} \mathrm{H}_{65} \mathrm{O}_{3} 569.4934$, found 569.4951 .

\section{8 (3R)-((1S,2R)-1,2-O-isopropylidene-cyclohex-3-ene-4-yl)oxy)-cholestane (23)}

Nitrogen was bubbled through a solution of enol ether $22(200 \mathrm{mg}, 0.351 \mathrm{mmol})$ in anhydrous benzene (12 mL) for $30 \mathrm{~min}$. Grubbs (11) catalyst (104 $\mathrm{mg}, 0.122 \mathrm{mmol})$ was then introduced the reaction mixture heated under nitrogen at $60{ }^{\circ} \mathrm{C}$ for $1 \mathrm{~h}$. Additional catalyst (52 $\mathrm{mg}, 0.061 \mathrm{mmol}$ ) was then added and heating continued for $1 \mathrm{~h}$, at which time, the

solvent was removed under reduced pressure. FCC of the residue provided $23(150 \mathrm{mg}, 78 \%)$ as a light brown oil: $\mathrm{R}_{\mathrm{f}}=0.6$ (on alumina, $10 \%$ EtOAc: petroleum ether: $2 \%$ TEA); ${ }^{1} \mathrm{H}$ NMR 
$\left(500 \mathrm{MHz}, \mathrm{C}_{6} \mathrm{D}_{6}\right) \delta 0.66(\mathrm{dt}, 1 \mathrm{H}, \mathrm{J}=3.6,11.5 \mathrm{~Hz}), 0.77(\mathrm{~s}, 3 \mathrm{H}), 0.80(\mathrm{~s}, 3 \mathrm{H}), 0.85-1.78(\mathrm{~m}$, 1H), 0.94-1.0 (m, 27H), $1.06(\mathrm{~d}$, buried, $\mathrm{J}=6.8 \mathrm{~Hz}, 6 \mathrm{H}), 1.15,(\mathrm{~d}, 3 \mathrm{H}$, buried $\mathrm{J}=6.6 \mathrm{~Hz})$, $1.55(\mathrm{~s}$, buried, 3H), 1.71 (s, buried, 3H), $1.91(\mathrm{~m}, 1 \mathrm{H}), 1.97-2.15(\mathrm{~m}, 5 \mathrm{H}), 2.54(\mathrm{~m}, 1 \mathrm{H}), 4.08$ $(\mathrm{m}, 1 \mathrm{H}), 4.20(\mathrm{~m}, 1 \mathrm{H}), 4.81(\mathrm{t}, 1 \mathrm{H}, \mathrm{J}=5.1 \mathrm{~Hz}), 4.95(\mathrm{~d}, 1 \mathrm{H}, \mathrm{J}=3.9 \mathrm{~Hz}) ;{ }^{13} \mathrm{C}$ NMR $(125$ $\left.\mathrm{MHz}, \mathrm{C}_{6} \mathrm{D}_{6}\right) \delta 12.7,19.4,21.9,23.1,23.4,24.7,24.9,25.3,26.6,27.2,28.5,28.8,29.0,29.1$, 29.3, 32.8, 34.8, 36.0, 36.2, 36.6, 37.0, 37.4, 40.3, 40.8, 43.3, 45.1, 54.9, 57.1 (two peaks), 73.4, 74.2, 75.3, 94.1, 108.7, 157.3.

\section{9 $\quad$ B-carba-arabinoside (24-OAc)}

$\mathrm{BH}_{3} \cdot \mathrm{Me}_{2} \mathrm{~S}(0.20 \mathrm{~mL}$ of a $90 \%$ solution in dimethyl sulfide $)$ was added at $0{ }^{\circ} \mathrm{C}$, under a nitrogen atmosphere to a solution of $\mathbf{2 3}(120 \mathrm{mg}, 0.222 \mathrm{mmol})$ in THF $(7 \mathrm{~mL})$ and cooled to $0{ }^{\circ} \mathrm{C}$. The mixture was warmed to $\mathrm{rt}$, stirred for an additional $1 \mathrm{~h}$ at this temperature, then recooled to $0{ }^{\circ} \mathrm{C}$ and treated with a mixture of $3 \mathrm{~N} \mathrm{NaOH}(0.5 \mathrm{~mL})$ and $30 \%$ aqueous $\mathrm{H}_{2} \mathrm{O}_{2}$ $(0.5 \mathrm{~mL})$ for $30 \mathrm{~min}$. The mixture was then extracted with ether and the organic phase washed with saturated aqueous $\mathrm{NaHCO}_{3}$ and brine, dried $\left(\mathrm{Na}_{2} \mathrm{SO}_{4}\right)$, filtered and evaporated under reduced pressure. FCC of the residue provided 24 as a colorless oil $(100 \mathrm{mg}, 85 \%): \mathrm{R}_{\mathrm{f}}$ $=0.6\left(20 \%\right.$ EtOAc: petroleum ether); ${ }^{1} \mathrm{H}$ NMR $\left(500 \mathrm{MHz}, \mathrm{C}_{6} \mathrm{D}_{6}\right) \delta 0.70$ (bt, $1 \mathrm{H}, \mathrm{J}=11.0$ Hz), 0.81 (s, 3H), 0.85 (s, 3H), 0.90-1.84 (m, 30H), 1.05 (d, buried, 6H, J = 6.4 Hz), 1.15 (d, buried, 3H, J =6.2 Hz), 1.46 (s, buried, 3H), 1.63 (s, buried, 3H), $1.89(\mathrm{~m}, 1 \mathrm{H}), 2.02(\mathrm{~m}, 2 \mathrm{H})$, $2.09(\mathrm{~m}, 1 \mathrm{H}), 2.16(\mathrm{~m}, 1 \mathrm{H}), 2.81(\mathrm{~s}, 1 \mathrm{H}), 3.12(\mathrm{dt}, 1 \mathrm{H}, \mathrm{J}=4.0,9.8 \mathrm{~Hz}), 3.38(\mathrm{~m}, 1 \mathrm{H}), 3.93$ $(\mathrm{dd}, 1 \mathrm{H}, \mathrm{J}=7.5,9.8 \mathrm{~Hz}), 4.08(\mathrm{~m}, 1 \mathrm{H}), 4.12(\mathrm{dd}, 1 \mathrm{H}, \mathrm{J}=5.0,7.5 \mathrm{~Hz}) ;{ }^{13} \mathrm{C} \mathrm{NMR}(125 \mathrm{MHz}$, $\left.\mathrm{C}_{6} \mathrm{D}_{6}\right) \delta 12.2,12.3,18.8,21.4,22.6,22.8,24.0,24.2,24.4,24.7,26.5,28.2,28.4,28.5,29.0$, $30.0,32.3,35.3,35.6,35.7,36.0,36.5,37.3,39.7,40.3,42.7,44.9,54.6,56.5,56.6,73.7$, 
77.0 (two peaks), 77.8, 80.8, 108.6.

A portion of the material from the previous step $(40 \mathrm{mg}, 0.07 \mathrm{mmol})$ was dissolved in ethyl acetate $(2.0 \mathrm{~mL})$ and treated with acetic anhydride $(0.02 \mathrm{~mL}, 0.2 \mathrm{mmol})$ and DMAP $(8$ $\mathrm{mg}, 0.07 \mathrm{mmol})$ for $10 \mathrm{~min} . \mathrm{CH}_{3} \mathrm{OH}(0.1 \mathrm{~mL})$ was then added to the reaction mixture, and the solvent evaporated in vacuo. FCC of the residue afforded 24-OAc as a colorless oil (42 $\mathrm{mg}, 99 \%): \mathrm{R}_{\mathrm{f}}=0.7\left(15 \%\right.$ EtOAc: petroleum ether); $[\alpha]_{\mathrm{D}}{ }^{20}-13\left(\mathrm{c} 0.1, \mathrm{CHCl}_{3}\right) ;{ }^{1} \mathrm{H}$ NMR $(500$ $\left.\mathrm{MHz}, \mathrm{C}_{6} \mathrm{D}_{6}\right) \delta 0.72(\mathrm{bt}, 1 \mathrm{H}, \mathrm{J}=11.0 \mathrm{~Hz}), 0.80\left(\mathrm{~s}, 3 \mathrm{H}, \mathrm{CH}_{3}-18 / 19\right), 0.84\left(\mathrm{~s}, 3 \mathrm{H}, \mathrm{CH}_{3}-18 / 19\right)$, 1.05 (d, buried, $6 \mathrm{H}, \mathrm{J}=6.5 \mathrm{~Hz}, \mathrm{CH}_{3}-26,27$ ), 1.13 (d, buried, 3H, J = 6.5 Hz, $\mathrm{CH}_{3}-21$ ), 0.94$1.83(\mathrm{~m}, 30 \mathrm{H}), 1.42(\mathrm{~s}$, buried $3 \mathrm{H}), 1.80(\mathrm{~s}$, buried, 3H), 1.85-2.08 (m, 3H), $2.02(\mathrm{~s}$, buried, 3H), $2.14(\mathrm{~m}, 1 \mathrm{H}), 3.22$ (dt, 1H, J = 4.0, $10.2 \mathrm{~Hz}, \mathrm{H1}$ '), 3.37 (m, 1H, H3), 4.04 (m, 2H, H3',

4'), $5.64\left(\mathrm{dd}, 1 \mathrm{H}, \mathrm{J}=7.5,10.2 \mathrm{~Hz}, \mathrm{H} 2\right.$ '); ${ }^{13} \mathrm{C}$ NMR (125 MHz, $\left.\mathrm{C}_{6} \mathrm{D}_{6}\right) \delta 12.4$ (two peaks), $19.0,21.0,21.6,22.8,23.1,23.7,24.4,24.6,26.5,26.8,28.2,28.4,28.7,29.3,30.0,32.6$, 35.7, 35.9 (two peaks), 36.2, 36.7, 37.5, 39.9, 40.5, 42.9, 45.3, 54.8, 56.7, 56.8, 74.1, 75.9, 77.0, 78.3, 79.2, 109.4, 169.3. ESIHRMS $(\mathrm{M}+\mathrm{Na})^{+}$calculated for $\mathrm{C}_{38} \mathrm{H}_{64} \mathrm{O}_{5} \mathrm{Na}$ 623.4651, found 623.4642 .

\subsection{Cholestanyl $(4 R, 5 R)-4,5$-bis(benzyloxy)hept-6-enoate (25)}

The reaction of acid $\mathbf{1 9}(1.0 \mathrm{~g}, 2.94 \mathrm{mmol})$ and $\mathbf{2 0}(1.14 \mathrm{~g}, 2.94 \mathrm{mmol})$ following the esterification procedure described for the synthesis of $\mathbf{2 1}$, provided $25(1.80 \mathrm{~g}, 90 \%)$ as a colorless oil: $\mathrm{R}_{\mathrm{f}}=0.8\left(20 \%\right.$ EtOAc: petroleum ether); ${ }^{1} \mathrm{H}$ NMR $\left(500 \mathrm{MHz}, \mathrm{CDCl}_{3}\right) \delta 0.36$ (bt, 1H, J = 10.6 Hz), $0.53(\mathrm{~s}, 3 \mathrm{H}), 0.56(\mathrm{~s}, 3 \mathrm{H}), 0.62-0.1 .60(\mathrm{~m}, 27 \mathrm{H}), 0.81$ (d, buried, 6H, J $=6.2 \mathrm{~Hz}), 0.90(\mathrm{~d}, 3 \mathrm{H}, \mathrm{J}=6.0 \mathrm{~Hz}), 1.73-1.88(\mathrm{~m}, 4 \mathrm{H}), 2.04(\mathrm{~m}, 1 \mathrm{H}), 2.40(\mathrm{~m}, 2 \mathrm{H}), 3.50(\mathrm{~m}$, $1 \mathrm{H}), 3.78(\mathrm{t}, 1 \mathrm{H}, \mathrm{J}=6.9 \mathrm{~Hz}), 4.30(\mathrm{ABq}, 2 \mathrm{H}, \delta \Delta=0.26 \mathrm{ppm}, \mathrm{J}=12.0 \mathrm{~Hz}), 4.50(\mathrm{ABq}, 2 \mathrm{H}$, 
$\delta \Delta=0.23$ ppm, $\mathrm{J}=11.5 \mathrm{~Hz}), 4.79(\mathrm{~m}, 1 \mathrm{H}), 5.00(\mathrm{~d}, 1 \mathrm{H}, \mathrm{J}=10.5 \mathrm{~Hz}), 5.08(\mathrm{~d}, 1 \mathrm{H}, \mathrm{J}=17.0$

$\mathrm{Hz}), 5.65(\mathrm{~m}, 1 \mathrm{H}), 6.97-7.10(\mathrm{~m}, 6 \mathrm{H}), 7.22(\mathrm{~m}, 4 \mathrm{H}) ;{ }^{13} \mathrm{C} \mathrm{NMR}\left(125 \mathrm{MHz}, \mathrm{CDCl}_{3}\right) \delta 12.3$ (two peaks), 19.0, 21.5, 22.8, 23.0, 24.4, 24.5, 26.8, 28.0, 28.4, 28.7, 28.9, 31.2, 32.3, 34.6, 35.6, 35.7, 36.2, 36.7, 37.0, 39.9, 40.4, 42.9, 44.7, 54.4, 56.7 (two peaks), 70.8, 73.4, 73.5, $80.4,82.9,118.5,127.6$ (two peaks), 127.9, 128.1, 128.3, 128.5, 128.6, 135.5, 139.3, 139.6, 172.8; ESIHRMS (M+Na) ${ }^{+}$calculated for $\mathrm{C}_{48} \mathrm{H}_{70} \mathrm{O}_{4} \mathrm{Na} 733.5160$, found 733.5172.

\subsection{1 (3R)-((5R,6R)-5,6-bis(benzyloxy)octa-1,7-dien-2-yl)oxy)-3-cholestane (26)}

A solution of titanium tetrachloride $\left(0.09 \mathrm{~mL}, 2 \mathrm{M}\right.$ in $\left.\mathrm{CH}_{2} \mathrm{Cl}_{2}, 0.176 \mathrm{mmol}\right)$ was added to THF $(3 \mathrm{~mL})$ at $0{ }^{\circ} \mathrm{C}$. The mixture was stirred for $30 \mathrm{~min}$ at which point TMEDA (0.05 mL, $0.363 \mathrm{mmol})$ was added in one portion. The resulting yellow-brown suspension was allowed to warm to $\mathrm{rt}$ and stirred for $30 \mathrm{~min}$. At this point, zinc dust $(0.02 \mathrm{~g}, 0.03 \mathrm{mmol})$ and lead (11) chloride $(0.3 \mathrm{mg}, 1.0 \mu \mathrm{mol})$ were added in one portion, and stirring was continued at $\mathrm{rt}$ for $10 \mathrm{~min}$. A solution of $25(110 \mathrm{mg}, 0.155 \mathrm{mmol})$ and dibromomethane $(0.05 \mathrm{ml})$ in THF $(1 \mathrm{~mL})$ was then added via cannula to the reaction flask. The mixture was stirred at $60{ }^{\mathrm{O}} \mathrm{C}$ for $1 \mathrm{~h}$, cooled to $0{ }^{\mathrm{O}} \mathrm{C}$, then quenched by addition of saturated aqueous $\mathrm{K}_{2} \mathrm{CO}_{3}(2 \mathrm{~mL})$. The resulting mixture was warmed to $\mathrm{rt}$ and stirred at this temperature for 30 min, then diluted with ether $(2 \mathrm{~mL})$, stirred vigorously for an additional $15 \mathrm{~min}$, and filtered through basic alumina using 3\% triethylamine-ether as the eluent. The greenish-blue residue was triturated with diethyl ether $(3-5 \mathrm{~mL})$ and the combined ethereal extract was concentrated in vacuo. FCC over basic alumina afforded 26 as a yellow oil (72 $\mathrm{mg}, 66 \%): \mathrm{R}_{\mathrm{f}}$ $=0.7(10 \%$ EtOAc: petroleum ether: $1 \%$ TEA $) ;{ }^{1} \mathrm{H}$ NMR $\left(500 \mathrm{MHz}, \mathrm{C}_{6} \mathrm{D}_{6}\right) \delta 0.65(\mathrm{bt}, 1 \mathrm{H}, \mathrm{J}$ $=10.8 \mathrm{~Hz}), 0.78(\mathrm{~s}, 3 \mathrm{H}), 0.82(\mathrm{~s}, 3 \mathrm{H}), 0.84-1.76(\mathrm{~m}, 27 \mathrm{H}), 1.05(\mathrm{~d}$, buried, $6 \mathrm{H}, \mathrm{J}=6.5 \mathrm{~Hz})$, 
$1.15(\mathrm{~d}, 3 \mathrm{H}, \mathrm{J}=6.5 \mathrm{~Hz}), 1.98-2.05(\mathrm{~m} \mathrm{2H}), 2.14(\mathrm{~m}, 2 \mathrm{H}), 2.28(\mathrm{~m}, 1 \mathrm{H}), 2.53(\mathrm{~m}, 1 \mathrm{H}), 2.65$ (m, 1H), $3.79(\mathrm{~m}, 1 \mathrm{H}), 4.08(\mathrm{~m}, 2 \mathrm{H}), 4.17(\mathrm{~s}, 1 \mathrm{H}), 4.22(\mathrm{~s}, 1 \mathrm{H}), 4.44(\operatorname{app~d}, 1 \mathrm{H}, \mathrm{J}=12.1$ Hz), $4.70(\mathrm{~m}, 2 \mathrm{H}), 4.91(\operatorname{app} \mathrm{d}, 1 \mathrm{H}, \mathrm{J}=11.6 \mathrm{~Hz}), 5.28(\mathrm{~d}, 1 \mathrm{H}, \mathrm{J}=10.5 \mathrm{~Hz}), 5.36(\mathrm{~d}, 1 \mathrm{H}, \mathrm{J}=$ 17.4 Hz), $5.97(\mathrm{~m}, 1 \mathrm{H}), 7.16-7.36(\mathrm{~m}, 6 \mathrm{H}, \mathrm{J}=7.1 \mathrm{~Hz}), 7.48(\mathrm{~m}, 4 \mathrm{H}) ;{ }^{13} \mathrm{C}$ NMR $(125 \mathrm{MHz}$, $\left.\mathrm{C}_{6} \mathrm{D}_{6}\right) \delta 12.3,12.4,19.0,21.6,22.8,23.0,24.4,24.6,27.9,28.4,28.7,29.0,29.3,32.4,34.5$, $35.7,35.9,36.2,36.7,37.1,39.9,40.5,42.9,45.0,54.6,56.7,56.8,70.8,73.3,75.4,80.8$, $81.5,82.9,118.2,127.5,127.6$ (two peaks), 127.7, 127.9, 128.1, 128.3, 128.4, 128.5, 128.6, 135.9, 139.4, 139.9, 161.8; ESILRMS $(\mathrm{M}+\mathrm{Na})^{+}$calculated $\mathrm{C}_{49} \mathrm{H}_{72} \mathrm{O}_{3} \mathrm{Na}$ 731.55, found 731.54 .

\section{$4.12(3 R)-((1 R, 2 R)-1,2-d i-O$-benzyl-cyclohex-3-ene-4-yl)oxy)-cholestane (27)}

Treatment of $26(65 \mathrm{mg}, 0.092 \mathrm{mmol})$ following the RCM procedure described for the synthesis of $\mathbf{2 3}$ provided $27(50 \mathrm{mg}, 80 \%)$ as a light brown oil: $\mathrm{R}_{\mathrm{f}}=0.8(20 \%$ EtOAc: petroleum ether: $1 \% \mathrm{TEA}) ;{ }^{1} \mathrm{H}$ NMR $\left(500 \mathrm{MHz}, \mathrm{C}_{6} \mathrm{D}_{6}\right) \delta 0.45(\mathrm{bt}, 1 \mathrm{H}, \mathrm{J}=10.5 \mathrm{~Hz}), 0.58(\mathrm{~s}$, 3H), $0.63(\mathrm{~s}, 3 \mathrm{H}), 0.64-1.60(\mathrm{~m}, 25 \mathrm{H}), 0.84(\mathrm{~d}$, buried, 6H, J = 6.5 Hz), 0.95 (d, buried, 3H, $\mathrm{J}=6.5 \mathrm{~Hz}), 1.66-1.83(\mathrm{~m}, 4 \mathrm{H}), 1.93(\mathrm{~m}, 3 \mathrm{H}), 2.10(\mathrm{~m}, 1 \mathrm{H}), 2.34(\mathrm{~m}, 1 \mathrm{H}), 3.68(\mathrm{~m}, 1 \mathrm{H})$, $3.93(\mathrm{~m}, 1 \mathrm{H}), 4.27(\mathrm{bt}, 1 \mathrm{H}, \mathrm{J}=4.2 \mathrm{~Hz}), 4.40(\mathrm{ABq}, 2 \mathrm{H}, \Delta \delta=05 \mathrm{ppm}, \mathrm{J}=12.0 \mathrm{~Hz}), 4.56$ $(\mathrm{ABq}, 2 \mathrm{H}, \Delta \delta=03 \mathrm{ppm}, \mathrm{J}=12.0 \mathrm{~Hz}), 4.86(\mathrm{~d}, 1 \mathrm{H}, \mathrm{J}=4.0 \mathrm{~Hz}), 7.03-7.14(\mathrm{~m}, 6 \mathrm{H}), 7.26(\mathrm{~d}$, $2 \mathrm{H}, \mathrm{J}=7.5 \mathrm{~Hz}), 7.32(\mathrm{~d}, 2 \mathrm{H}, \mathrm{J}=7.5 \mathrm{~Hz}) ;{ }^{13} \mathrm{C} \mathrm{NMR}\left(125 \mathrm{MHz}, \mathrm{C}_{6} \mathrm{D}_{6}\right) \delta 12.3,12.4,19.0$, 21.6, 22.8, 23.0, 24.3, 24.4, 24.6, 26.1, 28.1, 28.4, 28.7, 29.0, 32.4, 34.7, 35.7, 35.9, 36.2, $36.7,37.1,39.9,40.5,42.9,44.9,54.6,56.7,56.8,71.1,71.2,75.1,77.1,77.5,94.0,126.9$, $127.5,127.6,127.8,127.9,128.1,128.3$ 128.5, 128.9, 139.7, 140.1, 156.5; ESILRMS $(\mathrm{M}+\mathrm{Na})^{+}$calculated $\mathrm{C}_{47} \mathrm{H}_{68} \mathrm{O}_{3} \mathrm{Na} 703.52$, found 703.51 


\subsection{3 $\beta$-carba-xyloside (28-OAc)}

Application of the hydroboration oxidation that was used for the synthesis of $\mathbf{2 4}$, to $27(50 \mathrm{mg}, 0.073 \mathrm{mmol}$ ) and acetylation of the crude reaction product (following the procedure used for 24-OAc), afforded $\beta$-carba-xyloside 28-OAc as a colorless oil (43 $\mathrm{mg}$,

$80 \%$ over two steps): $\mathrm{R}_{\mathrm{f}}=0.8$ (20\% EtOAc: petroleum ether); ${ }^{1} \mathrm{H}$ NMR $\left(500 \mathrm{MHz}, \mathrm{CDCl}_{3}\right) \delta$ $0.51(\mathrm{~m}, 1 \mathrm{H}), 0.57$ (s, 3H, $\left.\mathrm{CH}_{3}-18 / 19\right), 0.68$ (s, buried 3H, $\left.\mathrm{CH}_{3}-18 / 19\right), 0.80$ (bd, buried, 6H, $\left.\mathrm{J}=6.8 \mathrm{~Hz}, \mathrm{CH}_{3}-26,27\right), 0.65-1.75(\mathrm{~m}, 31 \mathrm{H}), 0.82\left(\mathrm{~d}\right.$, buried $\left.3 \mathrm{H}, \mathrm{J}=6.8 \mathrm{~Hz}, \mathrm{CH}_{3}-21\right), 1.87$ (m, 2H), $1.90(\mathrm{~s}, 3 \mathrm{H}), 1.98(\mathrm{~m}, 1 \mathrm{H}), 3.17(\mathrm{~m}, 1 \mathrm{H}, \mathrm{H} 3), 3.23\left(\mathrm{~m}, 1 \mathrm{H}, \mathrm{H} 1^{\prime}\right), 3.33$ (t, 1H, J = 9.0 Hz, H3'), 3.43 (m, 1H, H4'), 4.58 (m, 3H, PhCH x 3), 4.80 (app d, 1H, J = 11.0 Hz, PhCH), $4.85\left(\mathrm{t}, 1 \mathrm{H}, \mathrm{J}=9.5 \mathrm{~Hz}, \mathrm{H} 2\right.$ '), $7.19-7.25(\mathrm{~m}, 10 \mathrm{H}, \mathrm{ArH}) ;{ }^{13} \mathrm{C} \mathrm{NMR}\left(125 \mathrm{MHz}, \mathrm{CDCl}_{3}\right) \delta 12.1$, $12.3,18.7,21.2,22.6,22.8,23.8,24.2,26.4,27.5,28.0,28.3,28.9,29.4,32.1,35.4,35.5$, $35.7,35.8,36.2,37.1,39.5,40.1,42.6,45.0,54.4,56.3,56.5,72.6,75.1,76.7,77.0,79.0$, $80.9,83.5,127.5,127.6,127.7,127.8$ (two peaks), 128.0, 128.3, 128.4 (two peaks), 138.7, 138.8, 170.0; ESIHRMS (M+Na) ${ }^{+}$calculated $\mathrm{C}_{49} \mathrm{H}_{72} \mathrm{O}_{5} \mathrm{Na} 763.5307$, found 763.5277.

\section{Acknowledgment}

This work was supported by the National Science Foundation (\#1301330). A Research Centers in Minority Institutions Program grant from the National Institute of Health Disparities (MD007599) of the National Institutes of Health (NIH), which supports the infrastructure at Hunter College, and a Clinical Translational Science Center award (TR000457) from the NIH are also acknowledged. We thank Dr. Matthew Devany and Ms. 
Rong Wang for help with NMR and mass spectrometry measurements, respectively.

\section{References}

1. La Ferla, B.; Airoldi, C.; Zona, C.; Orsato, A.; Cardona, F.; Merlo, S.; Sironi, E.; D’Orazio, G.; Nicotra, F. Nat. Prod. Rep. 2011, 28, 630-648.

2. Bhattacharya, C.; Yu, Z.; Rishel, M. J.; Hecht, S. M. Biochemistry 2014, 53, 32643266.

3. Wojtkielewicz, A.; D1ugosz,M.; Maj, J.; Morzycki, J. W.; Nowakowski, M.; Renkiewicz, J.; Strnad, M.; Swaczynová, J.; Wilczewska, A. Z.; Wójcik, J. J. Med. Chem. 2007, 50, 3667-3673.

4. Lorent, J. H.; Quetin-Leclercq, J.; Mingeot-Leclercq, M.-P. Org. Biomol. Chem. 2014, 12, 8803-8022.

5. Shukla, R. K.; Tiwari, A. Crit. Rev. Ther. Drug Carrier Syst. 2011, 28, 255-292.

6. El Alaoui, A.; Saha, N.; Schmidt, F.; Monneret, C.; Florent, J. C. Bioorg. Med. Chem. 2006, 14, 5012-5019.

7. Goff, R. D.; Thorson, J. S. Med. Chem. Commun. 2014, 5. 1036-1047.

8. Cai, H.; Wang, H.-Y. L.; Venkatadri, R.; Fu, D.-X.; Forman, M.; Bajaj, S. O.; Li, H.; O’Doherty, G. A.; Arav-Boger, R. ACS. Med. Chem. Lett. 2014, 5, 395-399.

9. $\quad$ Beale, T. M.; Taylor, M. S. Org. Lett. 2013, 15, 1358-1361.

10. Arjona, O.; Gómez, A. M.; López, J. C.; Plumet. J. Chem. Rev. 2007, 107, 19192036.

11. Alonso, M.; Cañada, F. J.; Jiménez-Barbero, J.; Solís, D.; Gabius, H.-J. Cheng, X.; Kumaran, G.; Mootoo, D.R. Eur. J. Org. Chem. 2004, 1604-1613.

12. Sakairi, N.; Kuzuhara, H. Tetrahedron Lett. 1982, 23, 5327-5330.

13. Ogawa, S.; Hirai, K.; Odagiri, T.; Matsunaga, N.; Yamazaki, T.; Nakajima, A. Eur.J. Org. Chem. 1998, 1099-1109.

14. Cheng, X.; Khan, N.; Kumaran, G.; Mootoo, D. R. Org. Lett. 2001, 3, 1323-1326. 
15. Podolak, I.; Galanty, A.; Sobolewska, D. Phytochem. Rev. 2010, 9, 425-474.

16. Mana, S.; Gao, W.; Zhang, Y.; Huang, L.; Liu, C. Fitoterapia 2010, 81, 703-714.

17. Dinda, B.; Debnathb, S.; Mohantaa, B. C.; Harigayac, Y. Chem. \& Biodiv. 2010, 7, 2327-2580.

18. Zhou, Y.; Garcia-Prieto, C.; Carney, D. A.; Xu, R.-H.; Pelicano, H.; Kang, Y.; Yu, W.; Lou, C.; Kondo, S.; Liu, j.; Harris, D. M.; Estrov, Z.; Keating, M. J.; Jin, Z.; Huang, P. J. Natl. Cancer Inst. 2005, 97, 1781-1785.

19. Guazzelli, L.; Ulc, R.; Rydner, L.; Oscarson, S. Org. Biomol. Chem. 2015, 13, 65986610 .

20. Postema, M. H. D.; Piper, J. L.; Betts, R. L. J. Org. Chem. 2005, 70, 829-836.

21. Okada, A.; Ohshima, T.; Shibasaki, M. Tetrahedron Lett. 2001, 42, 8023-8026.

22. Arisawa, M.; Theeraldanon, C.; Nishida, A.; Nakagawa, M. Tetrahedron Lett. 2001, $42,8029-8032$.

23. Aggarwal, V. K.; Daly, A. M. Chem. Commun. 2002, 2490-2491.

24. Smith III, A. B.; Kim, D.-S. Org. Lett. 2005, 7, 3247-3250.

25. Ceccon, J.; Danoun, G.; Greene, A. E.; Poisson, J.-F. Org. Biomol. Chem. 2009, 7. 2029-2031.

26. Baird, L. J.; Timmer, M. S. M.; Teesdale-Spittle, P. H.; Harvey, J. E. J. Org. Chem. 2009, 74, 2271-2277.

27. Prasad, K. R.; Penchalaiah, K. Tetrahedron 2011, 67, 4268-4276.

28. Hyldtoft, L.; Madsen, R. J. Am. Chem. Soc. 2000, 122, 8444-8452.

29. Gurjar, M. K.; Nagaprasad, R.; Ramana, C. V. Tetrahedron Lett. 2002, 43, 75777579 .

30. Pine, S. H.; Pettit, R. J.; Geib, G. D.; Cruz, S. G.; Gallego, C. H.; Tijerina, T.; Pine, R. D. J. Org. Chem. 1985, 50, 1212-1216.

31. Takai, K.; Kakiuchi, T.; Kataoka, Y.; Utimoto, K.; J. Org. Chem. 1994, 59, 26682670. 